PROCEEDINGS OF THE

AMERICAN MATHEMATICAL SOCIETY

Volume 139, Number 3, March 2011, Pages 1099-1104

S 0002-9939(2010)10534-8

Article electronically published on August 10, 2010

\title{
ISOMORPHISMS OF SPACES OF CONTINUOUS AFFINE FUNCTIONS ON COMPACT CONVEX SETS WITH LINDELÖF BOUNDARIES
}

\author{
PAVEL LUDVÍK AND JIŘÍ SPURNÝ
}

(Communicated by Nigel J. Kalton)

\begin{abstract}
Let $X, Y$ be compact convex sets such that every extreme point of $X$ and $Y$ is a weak peak point and both ext $X$ and ext $Y$ are Lindelöf spaces. We prove that if there exists an isomorphism $T: \mathfrak{A}^{c}(X) \rightarrow \mathfrak{A}^{c}(Y)$ with $\|T\| \cdot\left\|T^{-1}\right\|<2$, then ext $X$ is homeomorphic to ext $Y$. This generalizes results of C. H. Chu and H. B. Cohen.
\end{abstract}

\section{INTRODUCTION}

If $X$ is a compact convex set in a real locally convex space, let $\mathfrak{A}^{c}(X)$ stand for the space of all continuous affine functions, $\mathfrak{A}^{b}(X)$ for the space of all bounded affine functions on $X$, and ext $X$ for the set of extreme points.

We refer the reader to [5, pp. 72,73,75] for notions of the theory of compact convex sets. We just mention that $X$ can be embedded to $\left(\mathfrak{A}^{c}(X)\right)^{*}$ via the evaluation mapping $\phi: X \rightarrow\left(\mathfrak{A}^{c}(X)\right)^{*}$ defined as $\phi(x)(f)=f(x), f \in \mathfrak{A}^{c}(X), x \in X$. The dual unit ball $B_{\left(\mathfrak{A}^{c}(X)\right)^{*}}$ equals the convex hull co $(X \cup-X)$, and $\left(\mathfrak{A}^{c}(X)\right)^{*}$ coincides with span $X$, the linear span of $X$. Further, any function $f \in \mathfrak{A}^{b}(X)$ has a unique extension to $\operatorname{span} X$, and this provides an identification of $\left(\mathfrak{A}^{c}(X)\right)^{* *}$ with $\mathfrak{A}^{b}(X)$.

For a set $F \subset X$, the complementary set $F^{\mathrm{cs}}$ is defined as the union of all faces of $X$ disjoint from $F$. A face $F$ of $X$ is said to be a split face if its complementary set $F^{\mathrm{cs}}$ is convex (and hence a face; see [1, p. 132]) and every point in $X \backslash\left(F \cup F^{\mathrm{cs}}\right.$ ) can be uniquely represented as a convex combination of a point in $F$ and a point in $F^{\mathrm{cs}}$.

We call $x \in \operatorname{ext} X$ a weak peak point if given $\varepsilon \in(0,1)$ and an open neighborhood $U$ of $x$, there exists $h \in \mathfrak{A}^{c}(X)$ such that $\|h\| \leq 1, h(x)>1-\varepsilon$ and $|h|<\varepsilon$ on $\operatorname{ext} X \backslash U$.

Received by the editors January 7, 2010 and, in revised form, April 9, 2010.

2010 Mathematics Subject Classification. Primary 46A55, 46E15, 54D20.

Key words and phrases. Compact convex set, extreme point, weak peak point, Lindelöf space, continuous affine function.

The first author was supported by grant GAČR 401/09/H007.

The second author was supported in part by the grants GAAV IAA 100190901 and GAČR 201/07/0388, and in part by the Research Project MSM 0021620839 from the Czech Ministry of Education.

(C)2010 American Mathematical Society Reverts to public domain 28 years from publication 
Let us also recall that any weak peak point of a compact convex set $X$ is a split face and the converse holds if ext $X$ is closed; see [5, Proposition 1].

The following results are proved in [5, Theorems 7 and 12] by C. H. Chu and H. B. Cohen:

Let $X$ and $Y$ be compact convex sets and let $T: \mathfrak{A}^{c}(X) \rightarrow \mathfrak{A}^{c}(Y)$ be an isomorphism satisfying $\|T\| \cdot\left\|T^{-1}\right\|<2$. If

- $X$ and $Y$ are metrizable and each point of ext $X$ and $\operatorname{ext} Y$ is a weak peak point, or

- the sets ext $X$ and ext $Y$ are closed and each extreme point of $X$ and $Y$ is a split face,

then the sets ext $X$ and ext $Y$ are homeomorphic.

The aim of our paper is to show that the method of the proof of [5, Theorem 7] is applicable in a more general setting that covers both results mentioned above.

Theorem 1.1. Let $X, Y$ be compact convex sets such that every extreme point of $X$ and $Y$ is a weak peak point and both $\operatorname{ext} X$ and ext $Y$ are Lindelöf spaces. Let $T: \mathfrak{A}^{c}(X) \rightarrow \mathfrak{A}^{c}(Y)$ be an isomorphism with $\|T\| \cdot\left\|T^{-1}\right\|<2$. Then ext $X$ is homeomorphic to ext $Y$.

As in [5. Corollaries 13 and 14], this yields a corollary for function algebras: Let $\mathcal{A}$ and $\mathcal{B}$ be function algebras with Lindelöf Choquet boundaries, and let $T: \operatorname{re} \mathcal{A} \rightarrow$ re $\mathcal{B}$ be an isomorphism satisfying $\|T\| \cdot\left\|T^{-1}\right\|<2$. Then the Choquet boundaries of $\mathcal{A}$ and $\mathcal{B}$ are homeomorphic.

We recall that the construction from [3, Section VII] (see also [1, Proposition I.4.15] or [2, Theorem 3.2.4]) yields an example of a non-metrizable simplex $X$ such that ext $X$ is a Lindelöf non-closed subset of $X$ and every extreme point of $X$ is a weak peak point. To see this, let $B \subset[0,1]$ be a Bernstein set (see $[9$, Theorem 5.3]) and let

$$
K=([0,1] \times\{0\}) \cup \bigcup_{x \in B}(\{x\} \times[0,1])
$$

be endowed with the "porcupine" topology (see [3, Section VII]). Precisely, if $x \in B$ and $t \in(0,1]$, then a basis of neighborhoods of $(x, t)$ consists of sets of the form $\{x\} \times U$, where $U \subset[0,1]$ is a neighborhood of $t$. If $x \in[0,1]$, then a basis of neighborhoods of $(x, 0)$ consists of sets of the form

$$
(U \times\{0\}) \cup\left((U \times[0,1]) \backslash \bigcup_{i=1}^{n}\left(\left\{x_{i}\right\} \times F_{i}\right)\right),
$$

where $n \in \mathbb{N}, U \subset[0,1]$ is a neighborhood of $x, x_{1}, \ldots, x_{n}$ are points in $B \cap U$ and $F_{1}, \ldots, F_{n}$ are compact subsets of $(0,1]$.

If $\lambda$ stands for Lebesgue measure on $[0,1]$, let

$$
H=\left\{f \in \mathcal{C}(K): f(x, 0)=\int_{[0,1]} f(x, t) d \lambda(t), x \in B\right\}
$$

and

$$
X=\left\{s \in H^{*}: s \geq 0, s(1)=1\right\} .
$$

Then $X$ endowed with the weak ${ }^{*}$ topology is a simplex and ext $X$ is homeomorphic to $(([0,1] \backslash B) \times\{0\}) \cup(K \backslash([0,1] \times\{0\}))$. It is easy to see that ext $X$ is a Lindelöf non-closed set and every extreme point of $X$ is a weak peak point. 
Example 1 on [5, p. 83] shows that Theorem 1.1 need not hold even for compact convex sets in finite dimensional spaces if we omit the assumption that extreme points are weak peak points. An example due to H. U. Hess (see [7]) shows that for every $\varepsilon>0$ there exist metrizable simplices $X, Y$ and an isomorphism $T: \mathfrak{A}^{c}(X) \rightarrow$ $\mathfrak{A}^{c}(Y)$ such that $\|T\| \cdot\left\|T^{-1}\right\|<1+\varepsilon$ and $\operatorname{ext} X$ is not homeomorphic to ext $Y$. Nevertheless, it is not clear whether Theorem 1.1 remains valid if we omit the topological assumption on the sets of extreme points.

Question 1.2. Let $X, Y$ be compact convex sets such that every extreme point of $X$ and $Y$ is a weak peak point and let $T: \mathfrak{A}^{c}(X) \rightarrow \mathfrak{A}^{c}(Y)$ be an isomorphism with $\|T\| \cdot\left\|T^{-1}\right\|<2$. Is it true that $\operatorname{ext} X$ is homeomorphic to ext $Y$ ?

We need to recall several notions not explained in [5]. If $X$ is a compact (Hausdorff) space, we write $\mathcal{C}(X)$ for the space of all continuous functions on $X$ and $\mathcal{M}^{1}(X)$ for the space of all probability Radon measures on $X$. (By a Radon measure we mean a complete measure that is inner regular with respect to compact sets and is defined on a $\sigma$-algebra including all Borel subsets of $X$. We refer the reader to [6, Section 416] for more information on Radon measures.) We always consider $\mathcal{M}^{1}(X)$ to be endowed with the weak* topology. We say that a function $f: X \rightarrow \mathbb{R}$ is universally measurable if $f$ is $\mu$-measurable for every $\mu \in \mathcal{M}^{1}(X)$.

If $X$ is a compact convex subset of a real locally convex space, any $\mu \in \mathcal{M}^{1}(X)$ has its unique barycenter $r(\mu) \in X$, i.e., the point $x \in X$ satisfying $f(x)=\mu(f)$ for any $f \in \mathfrak{A}^{c}(X)$. We sometimes say that $\mu$ represents $x$. A function $f: X \rightarrow \mathbb{R}$ is strongly affine (or satisfies the barycentric formula), if $f$ is universally measurable, $\mu(f)$ exists and $f(r(\mu))=\mu(f)$ for any $\mu \in \mathcal{M}^{1}(X)$. We write $\mathfrak{A}_{\mathrm{bf}}(X)$ for the space of all strongly affine functions on $X$ and recall that it is easy to see that any strongly affine function is bounded (see the proof of [8, Satz 2.1(c)]). We also recall that any semicontinuous affine function on $X$ is strongly affine; see [2, Theorem 1.6.1(ix)].

\section{Proof of Theorem 1.1}

The proof of the main theorem follows the idea of the proof of [5, Theorems 7 and 12]. Hence we recall the main steps of their proof and point out our modifications. We start the proof with a minimum principle which is crucial for us because then [10, Lemma 2.4] is applicable for functions $T^{* *} f, f \in \mathfrak{A}_{\mathrm{bf}}(X)$.

Lemma 2.1. Let $X$ be a compact convex set such that ext $X$ is Lindelöf. If $f \in$ $\mathfrak{A}_{\mathrm{bf}}(X)$ satisfies $|f(x)| \leq c$ for all $x \in \operatorname{ext} X$, then $|f(x)| \leq c$ for all $x \in X$.

Proof. Let $x \in X$ be given. We find a maximal measure $\mu \in \mathcal{M}^{1}(X)$ representing the point $x$ (see [2, Theorem 1.6.4]) and define

$$
A=\{y \in X:|f(y)| \leq c\} .
$$

Then $A$ is a $\mu$-measurable set, and we claim that $\mu(A)=1$.

Indeed, let $K \subset X$ be an arbitrary compact set disjoint from $A$. Since $A \supset \operatorname{ext} X$, for any $y \in \operatorname{ext} X$ we can find its closed neighborhood not intersecting $K$. The set $\operatorname{ext} X$ is Lindelöf, and thus we can select countably many closed sets $F_{n} \subset X$, $n \in \mathbb{N}$, such that

$$
\operatorname{ext} X \subset \bigcup_{n=1}^{\infty} F_{n} \subset X \backslash K
$$


By [4, Theorem 27.11], $\mu\left(\bigcup_{n=1}^{\infty} F_{n}\right)=1$, and hence $\mu(K)=0$. By the regularity of $\mu, \mu(X \backslash A)=0$, and hence

$$
|f(x)|=\left|\int_{X} f d \mu\right| \leq \int_{A}|f| d \mu \leq c .
$$

This concludes the proof.

Proof of Theorem 1.1. Let $T: \mathfrak{A}^{c}(X) \rightarrow \mathfrak{A}^{c}(Y)$ be an isomorphism satisfying $\|T\|$. $\left\|T^{-1}\right\|<2$. We assume that there exist $c, c^{\prime} \in \mathbb{R}$ such that $1<c<c^{\prime}<2$ and $\|T\|<2$ and $\|T f\| \geq c^{\prime}\|f\|$ for all $f \in \mathfrak{A}^{c}(X)$ (otherwise we would find $1<c<c^{\prime}<2$ such that $\|T\| \cdot\left\|T^{-1}\right\|<\frac{2}{c^{\prime}}<2$ and consider the mapping $c^{\prime}\left\|T^{-1}\right\| T$; see [5, p. 76]).

Claim 1. For any $f \in \mathfrak{A}^{b}(X)$ and $g \in \mathfrak{A}^{b}(Y)$ non-zero, $\left\|T^{* *} f\right\|>c\|f\|$ and $\left\|\left(T^{-1}\right)^{* *} g\right\|>\frac{1}{2}\|g\|$.

Proof of Claim 1. The first inequality follows from

$$
\|f\|=\left\|\left(T^{-1}\right)^{* *} T^{* *} f\right\| \leq\left(c^{\prime}\right)^{-1}\left\|T^{* *} f\right\|<c^{-1}\left\|T^{* *} f\right\| ;
$$

the second one is analogous.

If $x \in \operatorname{ext} X$, we recall that $\left(\mathfrak{A}^{c}(X)\right)^{*}=\operatorname{span}\{x\} \oplus_{\ell^{1}} \operatorname{span}\{x\}^{\text {cs }}$ because $\{x\}$ is a split face; see [5, p. 72]. Hence, given $y \in Y$, following [5, p. 76] we can write

$$
T^{*} y=\lambda x+\mu \quad \text { for some } \lambda \in \mathbb{R} \text { and } \mu \in \operatorname{span}\{x\}^{\mathrm{cs}} .
$$

It is proved in [5, p. 77] that $\|\mu\|<2-c$ whenever $y \in Y$ satisfies $|\lambda|>c$.

We recall the construction of mappings $\rho: Y \rightarrow \operatorname{ext} X$ and $\tau: X \rightarrow \operatorname{ext} Y$. Given $x \in \operatorname{ext} X$, we denote by $\chi_{\{x\}}$ the characteristic function of the set $\{x\}$. Then the upper envelope function $h_{x}=\widehat{\chi}_{\{x\}}$, defined as

$$
\widehat{\chi}_{\{x\}}(z)=\inf \left\{h(z): h \in \mathfrak{A}^{c}(X), h>\chi_{\{x\}}\right\} \quad \text { for } z \in X,
$$

is upper semicontinuous and affine (see [5, p. 73]), and thus strongly affine (see [2, Theorem 1.6.1(ix)]). By [5, p. 77], for each $y \in \operatorname{ext} Y$ there is at most one point $x \in \operatorname{ext} X$ such that $\left|T^{* *} h_{x}(y)\right|>c$. Let

$$
Y^{\prime}=\left\{y \in \operatorname{ext} Y: \text { there exists } x \in \operatorname{ext} X \text { with }\left|T^{* *} h_{x}(y)\right|>c\right\},
$$

and let $\rho: Y^{\prime} \rightarrow X$ be defined by the property that $\rho(y)$ equals the unique point $x \in \operatorname{ext} X$ satisfying $\left|T^{* *} h_{x}(y)\right|>c$.

Analogously, if

$$
X^{\prime}=\left\{x \in \operatorname{ext} X: \text { there exists } y \in \operatorname{ext} Y \text { with }\left|\left(T^{-1}\right)^{* *} h_{y}(x)\right|>\frac{1}{2}\right\},
$$

then $\tau: X^{\prime} \rightarrow$ ext $Y$ can be defined by the requirement that $\tau(x)$ is the unique $y \in \operatorname{ext} Y$ satisfying $\left|\left(T^{-1}\right)^{* *} h_{y}(x)\right|>\frac{1}{2}$.

Claim 2. For any $x \in \operatorname{ext} X, T^{* *} h_{x} \in \mathfrak{A}_{\mathrm{bf}}(Y)$.

Proof of Claim 2. Since $T: \mathfrak{A}^{c}(X) \rightarrow \mathfrak{A}^{c}(Y)$, we have $T^{*}: \operatorname{span} Y \rightarrow \operatorname{span} X$. If $f \in \mathfrak{A}^{b}(X)$ and $\tilde{f}$ is the linear extension of $f$ to span $X$, then $T^{* *} f=\tilde{f} \circ T^{*}$. Since $\|T\|<2$

$$
T^{*} Y \subset 2 B_{\left(\mathfrak{A}^{c}(X)\right)^{*}}=\operatorname{co}(2 X \cup-2 X) .
$$


The sets $2 X$ and $-2 X$ are affinely homeomorphic to $X$, and hence $\tilde{f}$ is strongly affine on both of them. By [10, Lemma 2.4(b)],

$$
\tilde{f} \in \mathfrak{A}_{\mathrm{bf}}\left(2 B_{\left(\mathfrak{A}^{c}(X)\right)^{*}}\right)=\mathfrak{A}_{\mathrm{bf}}(\operatorname{co}(2 X \cup-2 X)) .
$$

Since $Y$ is affinely homeomorphic to $T^{*} Y$ and $T^{* *} f=\tilde{f} \circ T^{*}$, we obtain that $T^{* *} f \in \mathfrak{A}_{\mathrm{bf}}(Y)$.

Claim 3. The mappings $\rho: Y^{\prime} \rightarrow \operatorname{ext} X$ and $\tau: X^{\prime} \rightarrow \operatorname{ext} Y$ are surjective.

Proof of Claim 3. Let $x \in \operatorname{ext} X$ be given and assume that $\left|T^{* *} h_{x}(y)\right| \leq c$ for all $y \in \operatorname{ext} Y$. By Claims 1, 2 and Lemma 2.1, $\left|T^{* *} h_{x}\right| \leq c$ on $Y$. Then

$$
c \geq\left\|T^{* *} h_{x}\right\|>c\left\|h_{x}\right\|=c
$$

gives a contradiction. Hence $\rho$ is surjective.

Analogously, using the second part of Claim 1 we obtain that $\tau$ is surjective.

The following claim is essentially Lemma 6 of [5]. However, we recall its proof since it uses Lemma 2.1.

Claim 4. We have $X^{\prime}=\operatorname{ext} X$ and $Y^{\prime}=\operatorname{ext} Y$ and, for any $x \in \operatorname{ext} X$ and $y \in \operatorname{ext} Y, \rho(\tau(x))=x$ and $\tau(\rho(y))=y$.

Proof of Claim 4. We show that, for any $y \in Y^{\prime}$,

$$
\left|\left(T^{-1}\right)^{* *} h_{y}(\rho(y))\right|>\frac{1}{2} .
$$

Assuming $\left|\left(T^{-1}\right)^{* *} h_{y}(\rho(y))\right| \leq \frac{1}{2}$, Claim 2 and Lemma 2.1 yield

$$
d=\sup _{x \in \operatorname{ext} X}\left|\left(T^{-1}\right)^{* *} h_{y}(x)\right|=\sup _{x \in X}\left|\left(T^{-1}\right)^{* *} h_{y}(x)\right|=\left\|\left(T^{-1}\right)^{* *} h_{y}\right\| .
$$

By Lemma 2.1 and Claim $1, \frac{1}{2}<d$. Since $c>1$, we have $d>\frac{d}{c}$. Let $x^{\prime} \in \operatorname{ext} X$ be chosen such that

$$
\left|\left(T^{-1}\right)^{* *} h_{y}\left(x^{\prime}\right)\right|>\max \left\{\frac{d}{c}, \frac{1}{2}\right\}
$$

By the assumption, $\left|\left(T^{-1}\right)^{* *} h_{y}(\rho(y))\right| \leq \frac{1}{2}$, and thus $\rho(y) \neq x^{\prime}$.

By Claim 3 we can select $y^{\prime} \in Y^{\prime}$ with $\rho\left(y^{\prime}\right)=x^{\prime}$. Then $y^{\prime} \in\{y\}^{\text {cs }}$, and thus $h_{y}\left(y^{\prime}\right)=0$. If $T^{*} y^{\prime}=\lambda^{\prime} x^{\prime}+\mu^{\prime}, \lambda^{\prime} \in \mathbb{R}$ and $\mu^{\prime} \in \operatorname{span}\left\{x^{\prime}\right\}^{\text {cs }}$ (see (1)), then

$$
0=h_{y}\left(y^{\prime}\right)=\left(T^{-1}\right)^{* *} h_{y}\left(T^{*} y^{\prime}\right)=\left(T^{-1}\right)^{* *} h_{y}\left(\lambda^{\prime} x^{\prime}\right)+\left(T^{-1}\right)^{* *} h_{y}\left(\mu^{\prime}\right) .
$$

Since $\lambda^{\prime}=T^{* *} h_{x^{\prime}}\left(y^{\prime}\right)$, it follows from the definition of $\rho$ that $\left|\lambda^{\prime}\right|>c$.

Using this, (3) and (10) along with the subsequent remark, we obtain

$$
\begin{aligned}
d & <\left|\lambda^{\prime}\right| \frac{d}{c}<\left|\lambda^{\prime}\right|\left|\left(T^{-1}\right)^{* *} h_{y}\left(x^{\prime}\right)\right| \\
& =\left|\left(T^{-1}\right)^{* *} h_{y}\left(\lambda^{\prime} x^{\prime}\right)\right| \\
& =\left|\left(T^{-1}\right)^{* *} h_{y}\left(\mu^{\prime}\right)\right| \\
& \leq d\left\|\mu^{\prime}\right\|<d(2-c)<d .
\end{aligned}
$$

This contradiction yields the validity of (2).

Now, let $x \in \operatorname{ext} X$ be given. We find $y \in Y^{\prime}$ with $\rho(y)=x$. It follows from (2) that $x \in X^{\prime}$ and $\tau(x)=y$. Hence $X^{\prime}=\operatorname{ext} X$ and $\tau(\rho(y))=y$ for all $y \in Y^{\prime}$. 
If $y \in \operatorname{ext} Y$ is given, let $x \in \operatorname{ext} X$ be such that $\tau(x)=y$. If $y^{\prime} \in Y^{\prime}$ satisfies $\rho\left(y^{\prime}\right)=x$, from the previous argument we obtain

$$
y=\tau(x)=\tau\left(\rho\left(y^{\prime}\right)\right)=y^{\prime} .
$$

Hence $Y^{\prime}=\operatorname{ext} Y$ and it easily follows that $\rho(\tau(x))=x$ for any $x \in \operatorname{ext} X$.

By the proof of Theorem 7 on p. 78 in [5], the mappings $\rho$ and $\tau$ are continuous (we point out that this part of the argument is valid for arbitrary compact convex sets as mentioned in [5. p. 83]). This concludes the proof.

\section{ACKNOWLEDGMENT}

The authors would like to thank the referee for several useful comments leading to an improvement of the paper.

\section{REFERENCES}

[1] E. M. Alfsen. Compact convex sets and boundary integrals. Ergebnisse der Mathematik und ihrer Grenzgebiete, Band 57, Springer-Verlag, New York, 1971. MR0445271 (56:3615)

[2] L. Asimow and A. J. Ellis. Convexity theory and its applications in functional analysis, volume 16 of London Mathematical Society Monographs. Academic Press, Inc. [Harcourt Brace Jovanovich Publishers], London, 1980. MR623459 (82m:46009)

[3] E. Bishop and K. de Leeuw. The representations of linear functionals by measures on sets of extreme points. Ann. Inst. Fourier (Grenoble), 9:305-331, 1959. MR0114118(22:4945)

[4] G. Choquet. Lectures on analysis. Vols. I-III: Infinite dimensional measures and problem solutions. Edited by J. Marsden, T. Lance and S. Gelbart. W. A. Benjamin, Inc., New YorkAmsterdam, 1969.

[5] C. H. Chu and H. B. Cohen. Isomorphisms of spaces of continuous affine functions. Pacific J. Math., 155(1):71-85, 1992. MR 1174476 (93i:46041)

[6] D. H. Fremlin. Measure theory. Vol. 4. Torres Fremlin, Colchester, 2006. Topological measure spaces. Parts I, II. Corrected second printing of the 2003 original. MR 2462372

[7] H. U. Hess. On a theorem of Cambern. Proc. Amer. Math. Soc., 71(2):204-206, 1978. MR500490 (80e:46010)

[8] U. Krause. Der Satz von Choquet als ein abstrakter Spektralsatz und vice versa. Math. Ann., 184(4):275-296, 1970. MR.1513280

[9] John C. Oxtoby. Measure and category. A survey of the analogies between topological and measure spaces. Graduate Texts in Mathematics, Vol. 2, Springer-Verlag, New York, 1971. MR0393403 (52:14213)

[10] J. Spurný. Baire classes of Banach spaces and strongly affine functions. Trans. Amer. Math. Soc., 362(3):1659-1680, 2010. MR2563744

Department of Mathematical Analysis, Faculty of Mathematics and Physics, Charles University, Sokolovská 83, 18675 Praha 8, Czech Republic

E-mail address: ludvik@karlin.mff.cuni.cz

Department of Mathematical Analysis, Faculty of Mathematics and Physics, Charles University, Sokolovská 83, 18675 Praha 8, Czech Republic

E-mail address: spurny@karlin.mff.cuni.cz 